Network Working Group

Request for Comments: 4710

Category: Standards Track
A. Siddiqui

D. Romascanu

Avaya

E. Golovinsky

Alert Logic

October 2006

\title{
Real-time Application Quality-of-Service Monitoring (RAQMON) Framework
}

Status of This Memo

This document specifies an Internet standards track protocol for the Internet community, and requests discussion and suggestions for improvements. Please refer to the current edition of the "Internet Official Protocol Standards" (STD 1) for the standardization state and status of this protocol. Distribution of this memo is unlimited.

Copyright Notice

Copyright (C) The Internet Society (2006).

\section{Abstract}

There is a need to monitor end-devices such as IP phones, pagers, Instant Messaging clients, mobile phones, and various other handheld computing devices. This memo extends the remote network monitoring (RMON) family of specifications to allow real-time quality-of-service (QOS) monitoring of various applications that run on these devices and allows this information to be integrated with the RMON family using the Simple Network Management Protocol (SNMP). This memo defines the framework, architecture, relevant metrics, and transport requirements for real-time QoS monitoring of applications.

Table of Contents

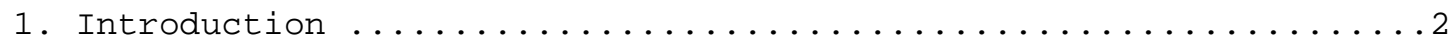

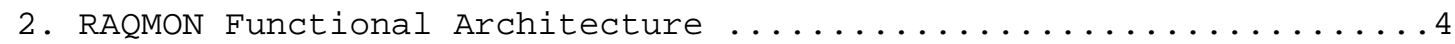

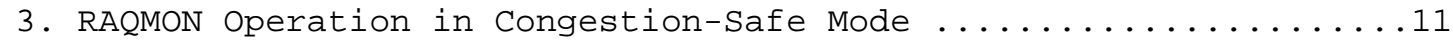

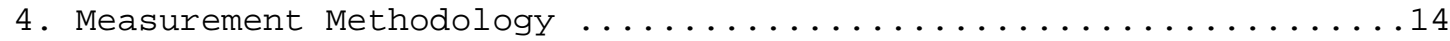

5. Metrics Pre-Defined for the BASIC Part of the RAQMON PDU .....14

6. Report Aggregation and Statistical Data processing ..........28

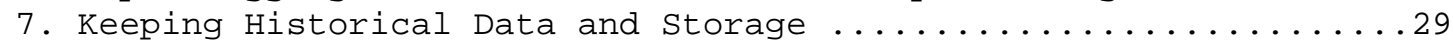

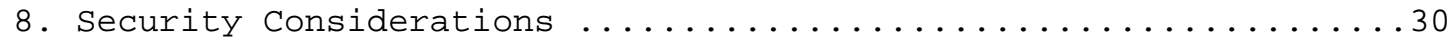

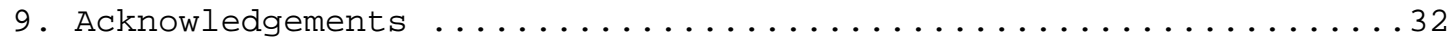

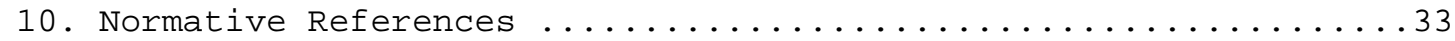

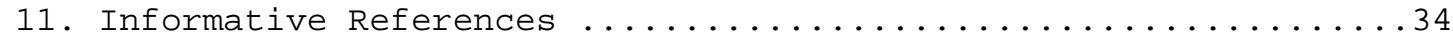




\section{Introduction}

With the growth of the Internet and advancements in embedded technologies, smart IP devices (such as IP phones, pagers, instant message clients, mobile phones, wireless handhelds, and various other computing devices) have become an integral part of our day-to-day operations. Enterprise operators, information technology (IT) managers, application service providers, network service providers, and so on, need to monitor these application and device types in order to ensure that end user quality-of-service (QOS) objectives are met. This memo describes a monitoring solution for these environments, extending the remote network monitoring (RMON) family of specifications [RFC2819]. These extensions support real-time QoS monitoring of typical applications that run on end-devices mentioned above, and they allow this information to be integrated using the familiar RMON family of specifications via SNMP [RFC3416].

The Real-time Application QoS Monitoring Framework (RAQMON) allows end-devices and applications to report QoS statistics in real time. Many real-time applications (as well as non-real-time applications managed within the RMON family of specifications) can report application-level QoS statistics in real time using the RAQMON Framework outlined in this memo. Some possible applications scenarios include applications such as Voice over IP, Fax over IP, Video over IP, Instant Messaging (IM), Email, software download applications, e-business style transactions, web access from handheld computing devices, etc.

The user experience of an application running on an IP end-device depends upon the type of application the user is running and the surrounding resources available to that application. An end-to-end application QoS experience is a compound effect of various application-level transactions and available network and host resources. For example, the end-to-end user experience of a Voice over IP (VoIP) call depends on the total time required to set up the call as much as on media-related performance parameters such as endto-end network delay, jitter, packet loss, and the type of codec used in a call. The performance of a VoIP call is also influenced by behavior of network protocols like the Reservation Protocol (RSVP), explicit tags in differentiated services (DiffServ) [RFC2475] or IEEE 802.1 [IEEE802.1D] along with available host resources such as device CPU or memory utilized by other applications while the call is ongoing.

The end-to-end application quality of service (QoS) experience is application context sensitive. For example, the kinds of parameters reported by an IP telephony application may not really be needed for other applications such as Instant Messaging. The RAQMON Framework 
offers a mechanism to report the end-to-end QoS experience appropriate for a specific application context by providing mechanisms to report a subset of metrics from a pre-defined list.

In order to facilitate a complete end-to-end view, RAQMON correlates statistics that involve:

i. "User, Application, Session"-specific parameters (e.g., session setup time, session duration parameters based on application context).

ii. "IP end-device"-specific parameters during a session (e.g., CPU usage, memory usage).

iii. "Transport network"-specific parameters during a session (e.g., end-to-end delay, one-way delay, jitter, packet loss etc).

At any given point, the applications at these devices can correlate such diverse data and report end-to-end performance. The RAQMON Framework specified in this memo offers a mechanism to report such end-to-end QoS view and integrate such a view into the RMON family of specifications. In particular, the RAQMON Framework specifies the following:

a. A set of basic metrics sent as reports between the RAQMON entities using for transport existing Internet Protocols such as TCP or SNMP.

b. Requirements to be met by the underlying transport protocols that carry the RAQMON reports.

C. A portion of the Management Information Base (MIB) as an extension of the RMON MIB Modules for use with network management protocols in the Internet community.

This memo provides the RAQMON functional architecture, RAQMON entity definitions and requirements, requirements for the transport protocols, a set of metrics, and an information model for the RAQMON reports.

Supplementary memos will describe the mapping of the basic RAQMON metrics onto different transport protocols. For example, the RAQMON PDU [RFC4712] memo provides definitions of syntactical PDU structure and use case scenarios of transmission of such PDUs over the Transmission Control Protocol (TCP) and the Simple Network Management Protocol (SNMP). 
The RAQMON MIB [RFC4711] memo describes the Management Information Base (MIB) for use with the SNMP protocol in the Internet community. The document proposes an extension to the Remote Monitoring MIB [RFC2819] to accommodate RAQMON solutions.

The key words "MUST", "MUST NOT", "REQUIRED", "SHALL", "SHALL NOT", "SHOULD", "SHOULD NOT", "RECOMMENDED", "MAY", and "OPTIONAL" in this document are to be interpreted as described in [RFC2119].

2. RAQMON Functional Architecture

The RAQMON Framework extends the architecture created in the RMON MIB [RFC2819] by providing application performance information as experienced by end-users. The RAQMON architecture is based on three functional components named below:

- RAQMON Data Source (RDS)

- RAQMON Report Collector (RRC)

- RAQMON MIB Structure

A RAQMON Data Source (RDS) is a functional component that acts as a source of data for monitoring purposes. End-devices like IP phones, cell phones, and pagers, and application clients like instant messaging clients, soft phones in PCs, etc., are envisioned to act as RDSs within the RAQMON Framework. 


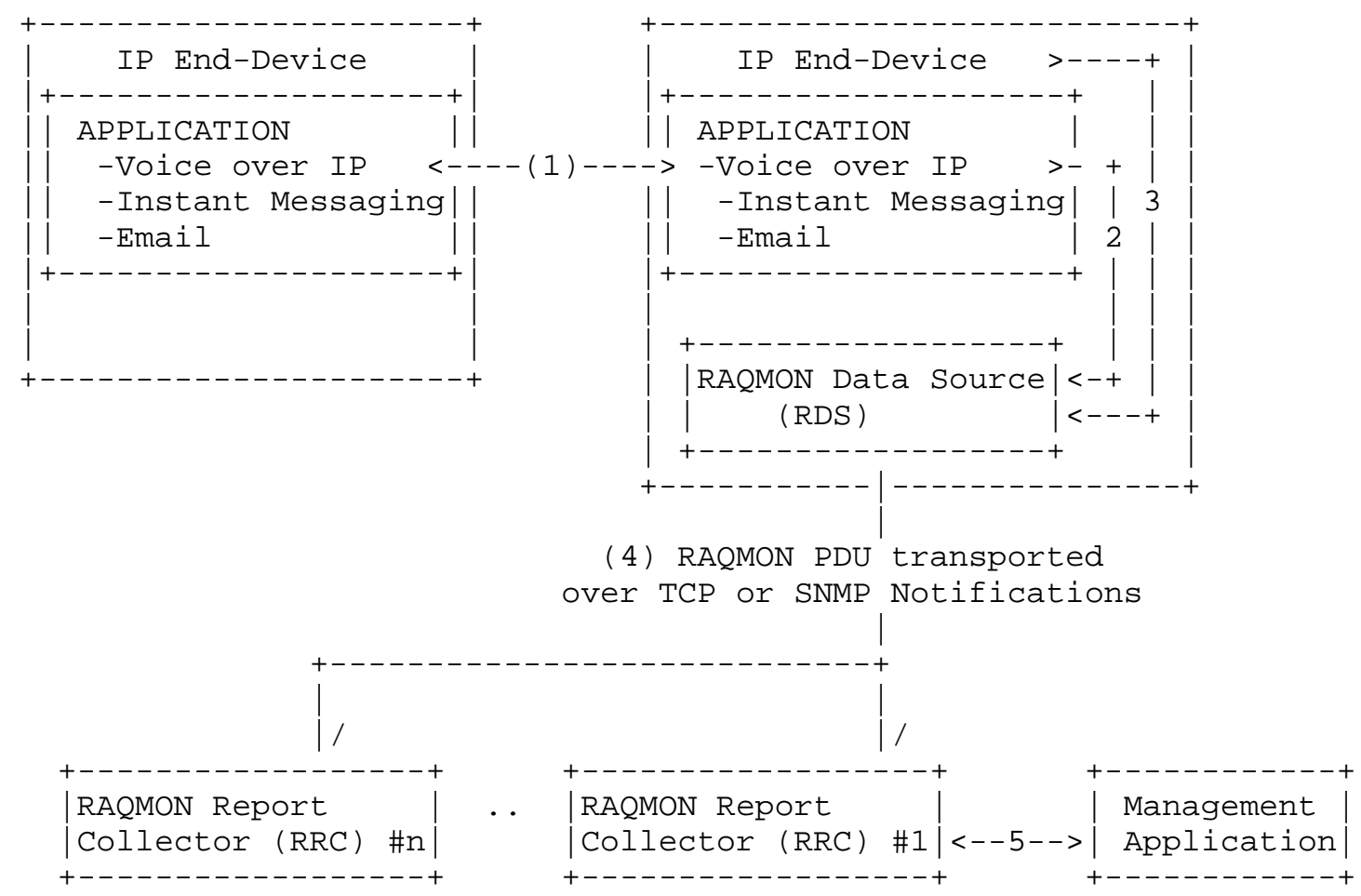

Figure 1 - RAQMON Framework.

(1) Communication Session between real-time applications

(2) Context-Sensitive Metrics

(3) Device State Specific Metrics

(4) Reporting session - RAQMON metrics transmitted over specified interfaces (Specific Protocol Interface, IP Address, port)

(5) Management Application - RRC interaction using the RAQMON MIB

A RAQMON Report Collector ( $R R C$ ) collects statistics from multiple RDSs, analyzes them, and stores such information appropriately. RRC is envisioned to be a network server, serving an administrative domain defined by the network administrator. The RRC component of the RAQMON architecture is envisioned to be computationally resourceful. Only RRCs should implement the RAQMON MIB module. 
The RAQMON Management Information Base (RAQMON MIB) extends the Remote Monitoring MIB [RFC2819] to accommodate the RAQMON Framework and exposes End-to-End Application QoS information to Network Management Applications.

\subsection{RAQMON Data Source (RDS)}

\subsubsection{RAQMON Data Source (RDS) Functional Architecture}

A RAQMON Data Source (RDS) is a source of data for monitoring purposes. The RDS monitoring function is performed in real time during communication sessions. The RDS entities capture QoS attributes of such communication sessions and report them within a RAQMON "reporting session".

An RDS is primarily responsible for abstracting IP end-devices and applications within the RAQMON architecture. It gathers the parameters for a particular communication session and forwards them to the appropriate RAQMON Report Collector (RRC). Since it is envisioned that the RDS functionality will be realized by writing firmware/software running on potentially small, low-powered enddevices, the design of the RDS element is optimized towards that end. Like the implementations of routing and management protocols, an implementation of RDS in an end-device will typically execute in the background, not in the data-forwarding path.

RDSs use a PUSH mechanism to report QoS parameters. While the applications running on the RDS decide about the content of the PDU appropriate for an application context, an RDS asynchronously sends out reports to $\mathrm{RRC}$.

The rate at which PDUs are sent from RDSs to RRCs is controlled by the applications' administrative domain policy. While this mechanism provides flexibility to gather a detailed end-to-end experience required by IT managers and system administrators, certain steps should be followed to operate RAQMON in congestion-safe manner. Section 3 addresses steps required for congestion-safe operation.

An RDS reports QoS statistics for simplex flows. At a given instance, a report from RDS is logically viewed as a collection of QoS parameters associated with a communication session as perceived by the reporting RDS. For example, if two IP phone users, Alice and John, are involved in a communication session, the end-to-end delay experienced by the IP phone user Alice could be different from the one experienced by the IP phone user John for a variety of reasons. Hence, a report from Alice's IP phone represents the QoS performance of that call as perceived by the RDS that resides in Alice's IP phone. 


\subsubsection{RAQMON Data Source (RDS) Requirements}

1. RAQMON Data Sources SHALL gather reports from multiple applications residing in that device and SHALL send out compound QoS reports associated with multiple communication sessions at a given moment.

Examples include a conference bridge hosting several different conference calls or a two-party video call consisting of audio/video sessions. In each case an RDS could send out one single RAQMON report that consists of multiple sub-reports associated with audio and video sessions or sub-reports for each conference call.

2. RAQMON Data Sources MUST implement the TCP transport and MAY implement the SNMP transport.

\subsubsection{Configuring RAQMON Data Sources}

In order to report statistics to RAQMON Report Collectors, RDSs will need to be configured with the following parameters:

1. The time interval between RAQMON PDUs. This parameter MUST be configured such that overflow of any RAQMON parameter within a PDU between consecutive transmissions is avoided.

2. The IP address and port of target RRC.

An RDS may use manual configuration for the RDS configuration parameters using command line interface (CLI), Telephone User Interface (TUI), etc.

One of the following mechanisms to gain access to configuration parameters can also be considered:

- RDS acts as a trivial file transfer protocol (TFTP) client and downloads text scripts to read the parameters.

- RDS acts as a Dynamic Host Configuration Protocol (DHCP) Client and gets RRC addressing information as a DHCP option.

- RDS acts as a DNS client and gets target collector information from a DNS Server.

- RDS acts as a LDAP Client and uses directory look-ups.

Identifying the DHCP option and structure to use, defining the structure of the configuration information in DNS, or defining a LDAP schema could be explored as items of future work. 
Compliance to the RAQMON specification does not require usage of any specific configuration mechanisms mentioned above. It is left to the implementers to choose appropriate provisioning mechanisms for a system.

\subsection{RAQMON Report Collector (RRC)}

\subsubsection{RAQMON Report Collector (RRC) Functional Architecture}

A RAQMON Report Collector (RRC) receives RAQMON PDUs from multiple RDSs and analyzes and stores the information in the RAQMON MIB. The $\mathrm{RRC}$ is envisioned to be computationally resourceful, providing a storage and aggregation point for a set of RDSs.

Since RDSs can belong to separate administrative domains, the RAQMON Framework allows RDSs to report QoS parameters to separate RRCs. Vendors can develop a management application to correlate information residing in different RRCs across multiple administrative domains to represent one communication session. However, such an applicationlevel specification is beyond the scope of this memo.

\subsubsection{RAQMON Report Collector (RRC) Requirements}

1. RAQMON Report Collectors MUST support the mandatory mapping over TCP of the RAQMON information model defined in [RFC4712] with the purpose of receiving RAQMON reports from RAQMON Data Sources (RDS).

2. RAQMON Report Collectors MAY support the optional mapping over SNMP notifications of the RAQMON information model defined in [RFC4712].

3. RAQMON Report Collectors MUST implement session timeout mechanisms to assume end of reporting for RDSs that have been out of reporting for a reasonable duration of time. Such timeout parameters SHOULD be configurable in vendor implementations, as programmable parameters at deployment.

4. RAQMON Report Collectors MUST support the RAQMON-MIB module and meet the compliance requirements of the raqmoncompliance MODULE-COMPLIANCE definition as described in [RFC4711]. The population of the RAQMON MIB with performance monitoring information is independent of the transport protocol, or protocols used to carry the information between RDSs and RRCs. 


\subsection{Information Model and RAQMON Protocol Data Unit (PDU)}

\subsubsection{RAQMON Information Model}

RAQMON defines a set of basic metrics that characterize the QoS of applications, as reported by RAQMON Data sources. This basic set of metrics is defined in section 5 of this memo. There is no minimal requirement for a mandatory set of metrics to be supported by an RDS.

Specific applications, new types of network appliances or new methods to measure and characterize the Qos of applications lead to the requirement for the information model to be extensible. To answer this need, the information model is designed so that vendors can extend it by adding new metrics.

Although NOT REQUIRED for RAQMON conformance, extensions of the information model can offer useful information for specific applications. An example of metrics that can extend the basic RAQMON information model are the detailed metrics for VoIP media monitoring and call quality included in the VoIP Metrics Report Block defined in [RFC3611].

The RAQMON Information model is expressed by defining a conceptual RAQMON Protocol Data Unit (PDU) .

\subsubsection{RAQMON Protocol Data Unit}

A RAQMON Protocol Data Unit (PDU) is a common data format understood by RDSs and RRCs. A RAQMON PDU does not transport application data but rather occupies the place of a payload specification at the application layer of the protocol stack. Different transport mappings may be used to carry RAQMON PDU between RDSs and RRCs. Transport protocol requirements are being defined in section 2.4 of this memo.

Though architected conceptually as a single PDU, the RAQMON PDU is functionally divided into two different parts. They are the BASIC part, and the Application-Specific Extensions, required for application-, vendor-, and device-specific extensions.

The BASIC part of the RAQMON PDU:

The BASIC part of the RAQMON PDU follows the SMI Network Management Private Enterprise Code 0 , indicating an IETF standard construct. The RAQMON PDU BASIC part offers an entry-type from a pre-defined list of QoS parameters defined in section 5 and allows applications to fill in appropriate values for those parameters. Application developers also have the flexibility to make an RDS report built only of a subset of the parameters listed in 
Section 5. There is no need to carry all metrics in every PDU; moreover, it is RECOMMENDED that static or pseudo-static metrics that do not change or seldom change for a given session or application will be send only when the session or application are initiated, and then at large time intervals.

The Application part of RAQMON PDU:

Since it is difficult to structure a BASIC part that meets the needs of all applications, RAQMON provides extension capabilities to convey application-, vendor-, and device-specific parameters for future use. Additional parameters can be defined within payload of the APP part of the PDU by the application developers or vendors. The owner of the definition of the application part of the RAQMON PDU is indicated by a vendor's SMI Network Management Private Enterprise Code defined in http://www.iana.org/assignments/enterprise-numbers. Such application-specific extensions should be maintained and published by the application vendor.

Though RDSs and RRCs are designed to be stateless for an entire reporting session, the framework requires an indication for the end of the reporting. For this purpose, an RDS MUST send a RAQMON NULL PDU. A NULL PDU is a RAQMON PDU containing ALL NULL values (i.e., nothing to report).

\subsection{RDS/RRC Network Transport Protocol Requirements}

The RAQMON PDUs rely on the underlying protocol(s) to provide transport functionalities and other attributes of a transport protocol, e.g., transport reliability, re-transmission, error correction, length indication, congestion safety, fragmentation/defragmentation, etc. The maximum length of the RAQMON data packet is limited only by the underlying protocols.

The following requirements MUST be met by the transport protocols:

1. The transport protocol SHOULD allow for RDS lightweight implementations. RDSs will be implemented on low-powered embedded devices with limited device resources.

2. Scalability - Since RRCs need to interact with a very large number (many tens, many hundreds, or more) of RDSs, scalability of the transport protocol is REQUIRED.

3. Congestion safety - as per [RFC2914]. See also section 3. 
4. Security - Since RAQMON statistics may carry sensitive system information requiring protection from unauthorized disclosure and modification in transit, a transport protocol that provides strong secure modes or allows for data encryption and integrity to be applied is REQUIRED.

5. NAT-Friendly - The transport protocol SHOULD comply with [RFC3235], so that an RDS could communicate with an RRC through a Firewall/Network Address Translation device.

6. The transport protocol MAY implement session timeout mechanisms to assume end of reporting for RDSs that have been out of reporting for a reasonable duration of time. Such timeout parameters SHOULD be configurable in vendor implementations, programmable at deployment.

7. Reliability - The RAQMON Framework expects PDUs to operate in lossy networks. However, retransmission is not included in the RAQMON framework, in order to keep the design simple. If retransmission is a necessity, RAQMON MAY operate over transport protocols, such as TCP.

In the future, if RAQMON PDUs are to be carried in an underlying protocol that provides the abstraction of a continuous octet stream rather than messages (packets), an encapsulation for the RAQMON packets must be defined to provide a framing mechanism. Framing is also needed if the underlying protocol contains padding so that the extent of the RAQMON payload cannot be determined. No framing mechanism is defined in this document. Carrying several RAQMON packets in one network or transport packet reduces header overhead.

Further memos like [RFC4712] describe how the PDU is transported over existing protocols like the Transmission Control Protocol (TCP) or the Simple Network Management Protocol (SNMP).

3. RAQMON Operation in Congestion-Safe Mode

RAQMON PDUs can be transmitted over multiple transport protocols. The RAQMON Framework will be congestion safe, if a RAQMON PDU is transported over TCP.

One solution to the congestion awareness problem could have been to discourage the use of UDP entirely for RAQMON. Though RAQMON PDUs can be transported over TCP, some transports like SNMP over TCP are not commonly practiced in practical deployments. 
The use of UDP inherently increases the risks of network congestion problems, as UDP itself does not define congestion prevention, avoidance, detection, or correction mechanisms. The fundamental problem with UDP is that it provides no feedback mechanism to allow a sender to pace its transmissions against the real performance of the network. While this tends to have no significant effect on extremely low-volume sender-receiver pairs, the impact of high-volume relationships on the network can be severe. This problem could be further aggravated by large RAQMON PDUs fragmented at the UDP level. Transport protocols such as DCCP can also be used as underlying RAQMON PDU transport, which provides flexibility of UDP style datagram transmission with congestion control.

It should be noted that the congestion problem is not just between RDS and RRC pairs, but whenever there is a high fan-in ratio, congestion could occur (e.g., many RDSs reporting to an RRC). Within the RAQMON Framework using UDP as a transport, congestion safety can be achieved in following ways:

1. Constant Transmission Rate: In a well-managed network, a constant transmission rate policy (e.g., 1 RAQMON PDU per device every $N$ seconds) will ensure congestion safety as devices are introduced into the network in a controlled manner. For example, in an enterprise network, IP Phones are added in a controlled manner, and a constant transmission rate policy can be sufficient to ensure congestion-safe operation. The configured rate needs to be related to the expected peak number of devices. As a worst-case scenario, if the RDSs enforce an administrative policy where the maximum PDU transmission rate is no more than one RAQMON PDU every two minutes, a UDP-based implementation can be as congestion safe as a TCP-based implementation. Such policies can be enforced while configuring RDSs, and the timers for the constant rate need to be randomly jittered.

2. Single outstanding requests: This approach requires that a request be sent at the application level, then there is a wait for some sort of response indicating that the request was received before sending anything else. This produces an effect described by some as "ping-ponging": traffic bounces back and forth between two nodes like a ping-pong ball in a match. Since there's only one ball in play between any two players at any given time, most of the potential for congestion cascades is eliminated. For reliability and efficiency reasons, this technique must include backed-off retransmissions. For example, if RAQMON PDUs are transported using SNMP INFORM PDUs over UDP, a SNMP response from the RRC SHOULD be processed by the RDS to implement this mechanism. [RFC4712] specifies that 
if the SNMP notifications transport mapping mechanism is implemented, it is RECOMMENDED to use INFORM PDUs, and it is NOT RECOMMENDED to use Trap PDUs.

This pacing or serialization approach has the side-effect of significantly reducing the maximum throughput, as transmission occurs in only one direction at a time and there is at least a 2xRTT (round-trip time) delay between transmissions. More sophisticated algorithms (such as those in TCP and stream Control Transmission Protocol (SCTP)) have been developed to address this, and it would be inappropriate to duplicate that work at the application level. Consequently, if greater efficiency is required than that provided by this simple approach, implementers SHOULD use TCP, SCTP, or another such protocol. But if one absolutely must use UDP, this approach works. It has been also used in other application scenarios like SIP over UDP.

3. By restricting transmission to a maximum transmission unit (MTU) size: An RDS may be faced with a request to deliver a large message using UDP as a transport. Fragmentation of such messages is problematic in several ways. Loss of any fragment requires time-out and retransmission of the message. The fragments are commonly transmitted out of the interface at local interface (usually LAN) rates, without awareness of the intervening network conditions. For these reasons, it is generally considered a bad practice to send large PDUs over UDP. If the MTU size is known, as an implementation, an RDS should not allow an application to send more information by limiting the size of transmissions over UDP to reduce the effects of fragmentation. As an alternate, an RDS MAY also send parameters to RRC over multiple RAQMON PDUs but identify them as part of the same RAQMON reporting session with exactly the same Network Time Protocol (NTP) [RFC1305] time stamp.

While the actual MTU of a link may not be known, common practice seems to indicate that the RDS local interface MTU is likely to be a reasonable "approximation". Where the actual path MTU is known, that value SHOULD be used instead.

4. Irrespective of choice of transport protocol, it is also RECOMMENDED that no more than 10\% network bandwidth be used for RDS/RRC reporting. More frequent reports from an RDS to RRC would imply requirements for higher network bandwidth usage. 
4. Measurement Methodology

It is not the intent of this document to recommend a methodology to measure any of the QoS parameters defined in section 5. Measurement algorithms are left to the implementers and equipment vendors to choose. There are many different measurement methodologies available for measuring application performance. These include probe-based, client-based, synthetic-transaction, and other approaches. This specification does not mandate a particular methodology and is open to any methodology that meets the minimum requirements. For conformance to this specification, it is REQUIRED that the collected data match the semantics described herein. However, it is RECOMMENDED that vendors use IETF-defined and International Telecommunication Union (ITU)-specified methodologies to measure parameters when possible.

5. Metrics Pre-Defined for the BASIC Part of the RAQMON PDU

The BASIC part of the RAQMON PDU provides for a list of pre-defined parameters frequently used by applications to characterize end-to-end application Quality of Service. This section defines a set of simple metrics to be contained in the BASIC part of the RAQMON PDU, through reference to existing IETF, ITU, and other standards organizations' documents. Appropriate IETF or ITU references are included in the metrics definitions.

As mentioned earlier, the RAQMON PDU also contains an applicationspecific part, where application- and vendor-specific information not included in BASIC part can be added as <Name, Value> pairs, or as a variable binding list. These extensions, managed independently by vendors or other organizations, should be published for wider interoperability.

Applications are not required to report all the parameters mentioned in this section, but should have the flexibility to report a subset of these parameters appropriate to an application context. The memo further identifies the parameters that RDSs are required to include in all PDUs for compliance, as well as optional parameters that RDSs may report as needed. The definitions presented here are meant to provide guidance to implementers, and IETF metric definition references are provided for each metric. Application developers should choose the metrics appropriate to their applications' needs. Syntactical representations of the parameters identified here are provided in the [RFC4712] specification. 


\subsection{Data Source Address (DA)}

The Data Source Address (DA) is the address of the data source. This could be either a globally unique IPv4 or IPv6 address, or a privately IPv4 allocated address as defined in [RFC1918].

It is expected that the DA would remain constant within a given communication session. RDSs SHOULD avoid sending these parameters within RAQMON reports too often to ensure an efficient usage of network resources.

\subsection{Receiver Address (RA)}

The Receiver Address (RA) takes the same form as the Data Source Address (DA) but represents the Receiver's Address. In a communication session, the reporting RDSs SHOULD fill in the other party's address as a Receiver Address. Like the Data Source Address, this could be either a globally unique IPv4 or IPv6 address, or a privately allocated IPv4 address as defined in [RFC1918].

It is expected that the Receiver Address (RA) would remain constant within a given communication session. RDSs SHOULD avoid sending these parameters within RAQMON reports too often in order to ensure an efficient usage of network resources.

\subsection{Data Source Name (DN)}

The Data Source Name (DN) item could be of various formats as needed by the application. Forms the DN could take include, but are not restricted to:

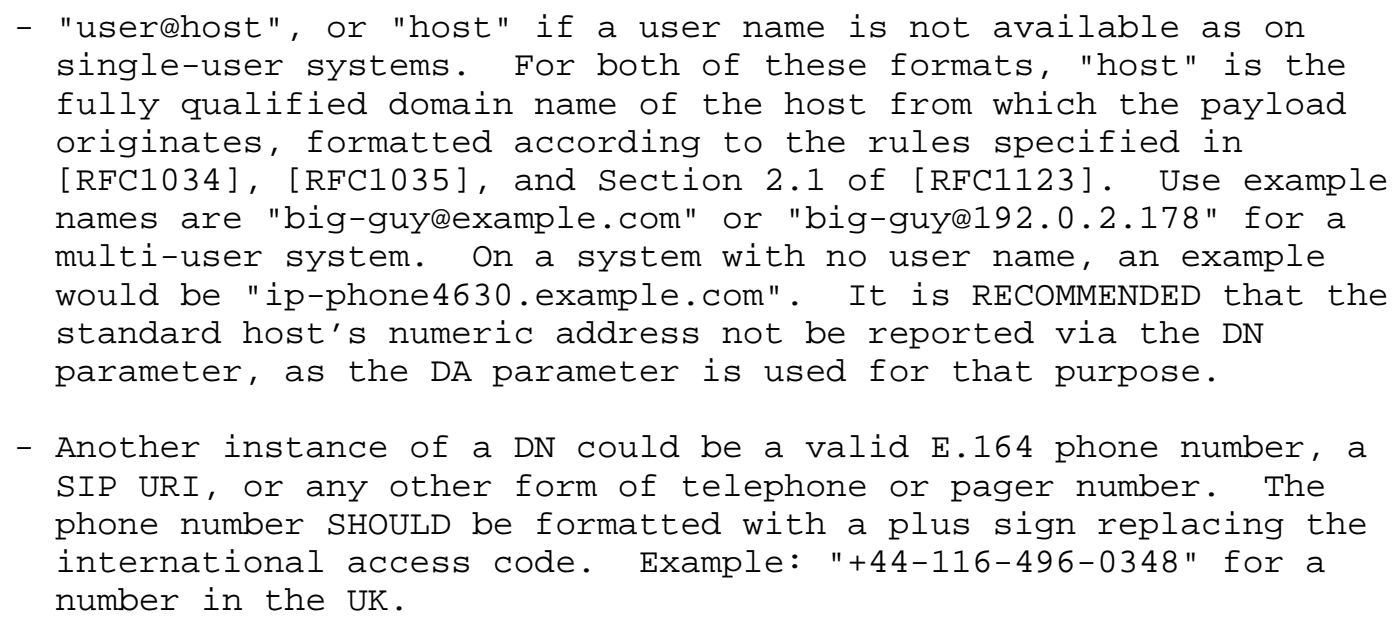


The DN value is expected to remain constant for the duration of a session. RDSs SHOULD avoid sending these parameters within RAQMON reports too often in order to ensure an efficient usage of network resources.

\subsection{Receiver Name (RN)}

The Receiver Name (RN) takes the same form as DN, but represents the Receiver's name. In a communication session, an application SHOULD supply as an RN the name of the other party with which it is communicating.

The RN value is expected to remain constant for the duration of a session. RDSs SHOULD avoid sending these parameters within RAQMON reports too often in order to ensure an efficient usage of network resources.

\subsection{Data Source Device Port Used}

This parameter indicates the source port used by the application for a particular session or sub-session in communication. Examples of ports include TCP Ports or UDP Ports, as used by communication application protocols such as Session Initiation Protocol (SIP), SIP for Instant Messaging and Presence Leveraging Extensions (SIMPLE), H.323, RTP, HyperText Transport Protocol (HTTP), and so on.

This parameter MUST be sent in the first RAQMON PDU.

\subsection{Receiver Device Port Used}

This parameter indicates the receiver port used by the application for a particular session or sub-session. Examples of ports include TCP Ports, or UDP Ports used by communication application protocols such as SIP, SIMPLE, H.323, RTP, HTTP, etc.

This parameter MUST be sent in the first RAQMON PDU.

\subsection{Session Setup Date/Time}

This parameter gives the time when the setup was initiated, if the application has a setup phase, or when the session was started, if such a setup phase does not exist. The time is represented using the timestamp format of the Network Time Protocol (NTP), which is in seconds relative to Oh UTC (Coordinated Universal Time) on 1 January 1900 [RFC1305].

This parameter SHOULD be sent only in the first RAQMON PDU, after the sesion setup is completed. 


\subsection{Session Setup Delay}

The Session setup Delay metric reports the time taken from an origination request being initiated by a host/endpoint to the media path being established (or a session progress indication being received from the remote host/endpoint), expressed in milliseconds. For example, in VoIP systems, a session setup time can be measured as the interval from the last DTMF (dual-tone multi-frequency) button pushed to the first ring-back tone that indicates that the far end is ringing. Another example would be the session setup Delay of a SIP call, which is measured as the elapsed time between when an INVITE is generated by a User Agent and when the 200 OK is received.

This parameter SHOULD be sent only in the first RAQMON PDU, after the session setup is completed.

\subsection{Session Duration}

The Session Duration metric reports how long a session or a subsession lasted. This metric is application context sensitive. For example, a VoIP Call Session Duration can be measured as the elapsed time between call pickup and call termination, including session setup time.

This parameter SHOULD be sent only in the first RAQMON PDU, after the session is terminated.

5.10. Session setup status

The Session setup status metric is intended to report the communication status of a session. Its values identify appropriate communication session states, such as Call Progressing, Call Established successfully, "trying", "ringing", "re-trying", "RSVP reservation failed", and so on.

Session setup status is meaningful in the context of applications. For this reason, applications SHOULD use this metric together with the application/name metrics defined in section 5.32.

This information could be used by network management systems to calculate parameters such as call success rate, call failure rate, etc., or by a debugging tool that captures the status of a call's setup phase as soon as a call is established.

This parameter SHOULD be sent after each change in the session status. 


\subsection{Round-Trip End-to-End Network Delay}

The Round-Trip End-to-End Network Delay, defined in [RFC3550] for applications running over RTP and in [RFC2681] for all other IP applications, is a key metric for Application QoS Monitoring. Some applications do not perform well (or at all) if the end-to-end delay between hosts is large relative to some threshold value. Erratic variation in delay values makes it difficult (or impossible) to support many real-time applications such as Voice over IP, Video over IP, Fax over IP etc.

The Round-Trip End-to-End Network delay of the underlying transport network is measured using methodologies described in [RFC3550] for RTP and in [RFC2681] for other IP applications.

Note that the packets used for measurement in some methodologies may be of a different type from those used for media (e.g., ICMP instead of RTP) and hence may differ in terms of route and queue priority. This may result in measured delays being different from those experienced on the media path. Conformance for this metric requires that actual application packets, or packets of the same application type, be used.

Support for RTP can be determined by the support of the RTP MIB [RFC2959] in the hosts running the applications or by inclusion of the string 'RTP' at the beginning of the Application Name (Section $5.32)$.

This parameter SHOULD be sent in each RAQMON PDU, if the RDS has the capability of determining its value and if the parameter is relevant for the application.

\subsection{One-Way End-to-End Network Delay}

The One-Way End-to-End Network Delay [RFC2679] metric reports the One-Way End-to-End delay encountered by traffic from the source to the destination network interface. One-Way Delay measurements identified by the IP Performance Metrics (IPPM) Working Group [RFC2679] will be used to measure one-way end-to-end network delay.

The need for such a metric is derived from the fact that the path from a source to a destination may be different from the path from the destination back to the source ("asymmetric paths"), such that different sequences of routers are used for the forward and reverse paths. Therefore, round-trip measurements actually measure the performance of two distinct paths together. 
Measuring each path independently highlights the performance

difference between the two paths that may traverse different Internet service providers, and even radically different types of networks (for example, research versus commodity networks, or ATM (Asynchronous Transfer Mode) versus Packet-over-SONET (Synchronous Optical) transport networks).

Even when the two paths are symmetric, they may have radically different performance characteristics due to asymmetric queuing. Performance of an application may depend mostly on the performance in one direction. For example, a file transfer using TCP may depend more on the performance in the direction that data flows than on the direction in which acknowledgements travel.

In QoS-enabled networks, provisioning in one direction may be radically different from provisioning in the reverse direction, and thus the QoS guarantees differ. Measuring the paths independently allows the verification of both guarantees.

RAQMON SHOULD NOT derive One-Way End-to-End Network Delay by assuming Internet paths are symmetric (i.e., dividing Round-Trip Delay by two) .

Note that the packets used for measurement in some methodologies may be of a different type from those used for media (e.g., ICMP instead of RTP) and hence may differ in terms of route and queue priority. This may result in measured delays being different from those experienced on the media path. Conformance for this metric requires that actual application packets, or packets of the same application type, be used.

This parameter SHOULD be sent in each RAQMON PDU, if the RDS has the capability of determining its value and if the parameter is relevant for the application.

\subsection{Application Delay}

Various Network Delay versions, as outlined in Sections 5.11 and 5.12, do not include delays associated with buffering, play-out, packet-sequencing, coding/decoding, etc., in the end-devices. The Application Delay metric defined in this section is targeted to capture all such delay parameters, providing a total application endpoint delay.

Application delay can be expressed as the time delay introduced between the network interface and the application-level presentation. Since it is difficult to envision usage of all sorts of applications, 
the following guidance is provided to the implementers to measure the application delay:

- The sending end contribution to application delay is defined as the sum of sample sequencing, accumulation, and encoding delay.

- The receiving end contribution to application delay is calculated as the sum of delays associated with buffering, play-out, packetsequencing, and decoding associated with the receiving direction, if relevant.

The endpoint application delay is defined as the sum of the receiving and sending contributions to delay measured or estimated within the endpoint that is generating this report.

It is easy to recognize that applications running on an IP device can experience same network delay but have different applicationassociated delay values. As such, the user experience associated with specific applications may vary while the network delay value remains same for both the applications.

Having network delay and application delay measurements available, a management application can represent the delay experienced by the end user at the application level as a sum of network delay and the application delays reported from the endpoints. However, the specification of such a management application is outside the scope of the RAQMON specification.

This parameter SHOULD be sent in each RAQMON PDU, if the RDS has the capability of determining its value and if the parameter is relevant for the application.

5.14. Inter-Arrival Jitter

The Inter-Arrival Jitter metric provides a short-term measure of network congestion [RFC3550]. The jitter measure may indicate congestion before it leads to packet loss. The inter-arrival jitter field is only a snapshot of the jitter at the time when a RAQMON PDU is generated and is not intended to be taken quantitatively as indicated in [RFC3550]. Rather, it is intended for comparison of inter-arrival jitter from one receiver over time. Such inter-arrival jitter information is extremely useful to understand the behavior of certain applications such as Voice over IP, Video over IP, etc. Inter-arrival jitter information is also used in the sizing of playout buffers for applications requiring the regular delivery of packets (for example, voice or video play-out). 
In [RFC3550], the selection function is implicitly applied to consecutive packet pairs, and the "jitter estimate" is computed by applying an exponential filter with parameter 1/16 to generate the estimate (i.e., j_new $=15 / 16 *$ j_old $+1 / 16 *$ j_new).

This parameter SHOULD be sent in each RAQMON PDU, if the RDS has the capability of determining its value and if the parameter is relevant for the application.

5.15. IP Packet Delay Variation

[RFC3393] provides guidance to several absolute jitter parameters. RAQMON uses the [RFC3393] definition of the IP Packet Delay Variation (ipdv) for packets inside a stream of packets. The IP Delay Variation metric is used to determine the dynamics of queues within a network (or router) where the changes in delay variation can be linked to changes in the queue length processes at a given link or a combination of links. Such a parameter provides visibility within an IP Network and a better understanding of application-level performance problems as it relates to IP Network performance.

This parameter SHOULD be sent in each RAQMON PDU, if the RDS has the capability of determining its value and if the parameter is relevant for the application.

\subsection{Total Number of Application Packets Received}

This metric reports the number of application payload packets received by the RDS as part of this session since the last RAQMON PDU was sent up until the time this RAQMON PDU was generated.

This parameter represents a very simple incremental counter that counts the number of "application" packets that an RDS has received. Application packets MAY include signaling packets. Since this count is a snapshot in time, depending on application type, it also varies based on the application states, e.g., an RDS within an application session will report the aggregated number of application packets that were sent out during signaling setup, media packets received, session termination, etc.

For example, during Voice over IP or Video over IP sessions setup, this counter represents the number of signaling-session-related packets that have been received that will be derived from the relevant application signaling protocol stack such as SIP or H.323, SIMPLE, and various other signaling protocols used by the application to establish the communication session. 
However, during a period when media is established between the communicating entities, this counter will be indicative of the number of RTP Frames that have been sent out to the communicating party since last PDU was sent out. The methodology described within RTCP $\mathrm{SR} / \mathrm{RR}$ reports [RFC3550] to count RTP frames will be applied wherever applications use RTP. This being a cumulative counter, applications need to take into consideration the possibility of the counter overflowing and restarting counting from zero.

Support for RTP can be determined by the support of the RTP MIB [RFC2959] in the hosts running the applications or by inclusion of the string 'RTP' at the beginning of the Application Name (Section 5.32 ).

This parameter SHOULD be sent in each RAQMON PDU, if the RDS has the capability of determining its value and if the parameter is relevant for the application.

5.17. Total Number of Application Packets sent

This metric reports the number of signaling and payload packets sent by the RDS as part of this session since the last RAQMON PDU was sent until the time this RAQMON PDU was generated. Applications packets MAY include signaling packets. Similar to the total number of application packets received parameter in section 5.16, this count is a snapshot in time. Depending on the application type, the counter also varies based on various application states, including packet counts for signaling setup, media establishment, session termination states, and so on. This being a cumulative counter, applications need to take into consideration the possibility of the counter overflowing and restarting counting from zero.

This parameter SHOULD be sent in each RAQMON PDU, if the RDS has the capability of determining its value and if the parameter is relevant for the application.

\subsection{Total Number of Application Octets Received}

This metric reports the total number of signaling and payload octets received in packets by the RDS as part of this session since the last RAQMON PDU was sent, up until the time this RAQMON packet was generated. Applications octets MAY include signaling octets. The methodology described by [RFC3550] will be applied wherever applications use RTP. This being a cumulative counter, applications need to take into consideration the possibility of the counter overflowing and restarting counting from zero. 
Support for RTP can be determined by the support of the RTP MIB [RFC2959] in the hosts running the applications or by inclusion of the string 'RTP' at the beginning of the Application Name (Section $5.32)$.

This parameter SHOULD be sent in each RAQMON PDU, if the RDS has the capability of determining its value and if the parameter is relevant for the application.

5.19. Total Number of Application Octets Sent

This metric reports the total number of signaling and payload octets received in packets by the RDS as part of this session since the last RAQMON PDU was sent, up until the time this RAQMON packet was generated. This is similar to the Total Number of Application Octets Received metric. Applications octets MAY include signaling octets. The methodology described by [RFC3550] will be applied wherever applications use RTP. This being a cumulative counter, applications need to take into consideration the possibility of the counter overflowing and restarting counting from zero.

Support for RTP can be determined by the support of the RTP MIB [RFC2959] in the hosts running the applications or by inclusion of the string 'RTP' at the beginning of the Application Name (Section $5.32)$.

This parameter SHOULD be sent in each RAQMON PDU, if the RDS has the capability of determining its value and if the parameter is relevant for the application.

\subsection{Cumulative Packet Loss}

The cumulative packet loss metric indicates the loss associated with the network as well as local device losses over time. This parameter is counted as the total number of application packets from the source that have been lost since the beginning of the session. This number is defined to be the number of packets expected less the number of packets actually received, where the number of packets received includes the count of packets that are late or duplicates. If a packet is discarded due to late arrival, then it MUST be counted as either lost or discarded but MUST NOT be counted as both.

Packet loss by the underlying transport network SHALL be measured using the methodologies described in [RFC3550] for RTP traffic and [RFC2680] for other IP traffic. The number of packets expected is defined to be the extended last sequence number received, as defined 
next, less the initial sequence number received. For RTP traffic, this may be calculated using techniques such as those shown in Appendix A.3 of [RFC3550].

Packet loss by the underlying transport network SHALL be measured using the methodologies described in [RFC3550] for RTP traffic and [RFC2680] for other IP traffic. The number of packets expected is defined to be the extended last sequence number received, as defined next, less the initial sequence number received. For RTP traffic, this may be calculated using techniques such as those shown in Appendix A.3 of [RFC3550].

Support for RTP can be determined by the support of the RTP MIB [RFC2959] in the hosts running the applications or by inclusion of the string 'RTP' at the beginning of the Application Name (Section $5.32)$.

This parameter SHOULD be sent in each RAQMON PDU, if the RDS has the capability of determining its value and if the parameter is relevant for the application.

\subsection{Packet Loss in Fraction}

The Packet Loss in Fraction metric represents the packet loss as defined above, but expressed as a fraction of the total traffic over time.

This parameter SHOULD be sent in each RAQMON PDU, if the RDS has the capability of determining its value and if the parameter is relevant for the application.

\subsection{Cumulative Application Packet Discards}

The RAQMON Framework allows applications to distinguish between packets lost by the network and those discarded due to jitter and other application-level errors. Though packet loss and discards have an equal effect on the quality of the application, having separate counts for packet loss and discards helps identify the source of quality degradation.

The packet discard metric indicates packets discarded locally by the device over time. Local device-level packet discard is captured as the total number of application-level packets from the source that have been discarded since the beginning of reception, due to late or early arrival, under-run or overflow at the receiving jitter buffer, or any other application-specific reasons. 
If the RDS cannot tell the difference between discards and lost packets, then it MUST report only lost packets and MUST NOT report discards.

This parameter SHOULD be sent in each RAQMON PDU, if the RDS has the capability of determining its value and if the parameter is relevant for the application.

\subsection{Packet Discards in Fraction}

The packet discards in fraction metric represents packets from the source that have been discarded since the beginning of the reception but expressed as a fraction of the total traffic over time.

This parameter SHOULD be sent in each RAQMON PDU, if the RDS has the capability of determining its value and if the parameter is relevant for the application.

\subsection{Source Payload Type}

The source payload type reports payload formats (e.g., media encoding) as sent by the data source, e.g., ITU G.711, ITU G.729B, H.263, MPEG-2, ASCII, etc. This memo follows the definition of Payload Type (PT) in [RFC3551]. For example, to indicate that the source payload type used for a session is PCMA (pulse-code modulation with A-law scaling), the value of the source payload field for the respective session will be 8 .

The source payload type value is expected to remain constant for the duration of a session, with the exception of events like dynamic codec changes. RDSs SHOULD avoid sending these parameters within RAQMON reports more often than necessary (e.g., at dynamic codec changes) to ensure an efficient usage of network resources.

If dynamic types (values 96 to 127, according to [RFC3551]) are being used to identify the source payload type, a RAQMON extension parameter MAY be defined to indicate the MIME subtypes. In the case where the RDS does send reports noting dynamic codec changes, there may be instances where this extension parameter is used only before or after the codec change, as the source payload may shift between the dynamic and static types.

\subsection{Receiver Payload Type}

The receiver payload type reports payload formats (e.g., media encodings) as sent by the other communicating party back to the source, e.g., ITU G.711, ITU G.729B, H.263, MPEG-2, ASCII, etc. This document follows the definition of payload type (PT) in [RFC3551]. 
For example, to indicate that the destination payload type used for a session is PCMA, the destination payload type field for the respective session will be 8 .

The destination payload type value is expected to remain constant for the duration of a session, with the exception of events like dynamic codec changes. RDSs SHOULD avoid sending these parameters within RAQMON reports more often than necessary (e.g., at dynamic codec changes) to ensure an efficient usage of network resources.

If dynamic types (values 96 to 127, according to [RFC3551]) are being used to identify the destination payload type, a RAQMON extension parameter MAY be defined to indicate the MIME subtypes. In the case where the RDS does send reports noting dynamic codec changes, there may be instances where this extension parameter is used only before or after the codec change, as the destination payload may shift between the dynamic and static types.

\subsection{Source Layer 2 Priority}

Many devices use Layer 2 technologies to prioritize certain types of traffic in the Local Area Network environment. For example, the 1998 Edition of IEEE 802.1D [IEEE802.1D], "Media Access Control Bridges", contains expedited traffic capabilities to support transmission of time-critical information. Many devices use that standard to mark Ethernet frames according to IEEE P802.1p standard. Details on these can be found in [IEEE802.1D], which incorporates P802.1p. The Source Layer 2 Priority RAQMON field indicates what Layer 2 values were used by the host running the RDS to prioritize these packets in the Local Area Network environment.

The Source Layer 2 Priority value is expected to remain constant for the duration of a session. Hosts running the RDSs SHOULD avoid sending these parameters within RAQMON reports too often in order to ensure an efficient usage of network resources.

\subsection{Source TOS/DSCP Value}

Various Layer 3 technologies are in place to prioritize traffic in the Internet. For example, the traditional IP Precedence [RFC791] and Type of Service (TOS) [RFC1812], or more recent technologies like Differentiated Services [RFC2474] [RFC2475], use the TOS octet in IPv4, whereas the traffic class octet is used to prioritize traffic in IPV6. Source Layer TOS/DCP RAQMON field reports the appropriate Layer 3 values used by the Data Source to prioritize these packets. 
The Source TOS/DSCP value is expected to remain constant for the duration of a session. Hosts running the RDSs SHOULD avoid sending these parameters within RAQMON reports too often in order to ensure an efficient usage of network resources.

5.28. Destination Layer 2 Priority

The Destination Layer 2 Priority reports the Layer 2 value used by the communication receiver to prioritize packets while sending traffic to the data source in the Local Area Networks environment. Like Source Layer 2 Priority, Destination Layer 2 Priority could indicate whether the destination has used Layer 2 technologies like IEEE P802.1p for priority queuing.

The Destination Layer 2 Priority value is expected to remain constant for the duration of a session. Hosts running the RDSs SHOULD avoid sending these parameters within RAQMON reports too often in order to ensure an efficient usage of network resources.

\subsection{Destination TOS/DSCP Value}

The Destination TOS/DSCP RAQMON field reports the values used by the Data Receiver to prioritize these packets received by the source. Similar to Source Layer 3 Priority, Destination Layer 3 Priority indicates whether the destination has used any Layer 3 technologies like IP Precedence [RFC791] and Type of Service (TOS) [RFC1812], or more recent technologies like Differentiated Service [RFC2474] [RFC2475].

The Destination TOS/DSCP value is expected to remain constant for the duration of a session. Hosts running the RDSs SHOULD avoid sending these parameters within RAQMON reports too often in order to ensure an efficient usage of network resources.

5.30. CPU Utilization in Fraction

This parameter captures the CPU usage of the hosts running the RDSs that may have very critical implications for gos of an end-device. It is computed as an average since the last reporting interval, and corresponds to the percentage of that time that the CPU was busy.

In the case of multiple CPU hosts, the maximum utilization among the different CPUS MUST be reported.

This parameter SHOULD be sent in each RAQMON PDU, if the RDS has the capability of determining its value and if the parameter is relevant for the application. 


\subsection{Memory Utilization in Fraction}

This parameter captures the memory usage of the hosts running the RDSs that may have very critical implications for QoS of an enddevice. It is computed as an average since the last reporting interval and corresponds to the average percentage of the total memory space critical for the applications in use during that time interval (e.g., primary CPU RAM, buffers).

In the case of multiple CPU hosts, the maximum memory utilization among the different CPUs MUST be reported.

This parameter SHOULD be sent in each RAQMON PDU, if the RDS has the capability of determining its value and if the parameter is relevant for the application.

\subsection{Application Name/Version}

The Application Name/Version parameter gives the name and, optionally, the version of the application associated with that session or sub-session, e.g., "XYZ VoIP Agent 1.2". This information may be useful for scenarios where the end-device is running multiple applications with various priorities and could be very handy for debugging purposes.

If the application is using RTP [RFC3550], the Application Name SHOULD begin with the string 'RTP'.

This parameter MUST be sent in the first RAQMON PDU.

6. Report Aggregation and Statistical Data processing

Within the RAQMON Framework, RRCs are expected to have significantly greater computational resources than RDSs. Consequently, various aggregation functions are performed by the RRCs, while RDSs are not burdened by statistical data processing such as computation of minima, maxima, averages, standard deviations, etc.

The RAQMON MIB provides minimal aggregation of the RAQMON parameters defined above. The RAQMON MIB is not designed to provide extensive aggregation like the Application Performance Measurement (APM) MIB [RFC3729] or the Transport Performance Metrics (TPM) MIB [RFC4150]. One should use APM and TPM MIBs to aggregate parameters based on protocols (e.g., performance of HTTP, RTP) or applications (e.g., performance of VoIP, Video Applications). 
In the RAQMON MIB, aggregation can be performed only on specific RAQMON metric parameters. Aggregation always results in statistical Mean/Min/Max values, according to these definitions:

Mean: Mean is defined as the statistical average of a metric over the duration of a communication session. For example, if an RDS reported End-to-End delay metric $N$ times within a communication session, then the Mean End-to-End Delay can be computed by summing of these $\mathrm{N}$ reported values, and then dividing by $\mathrm{N}$.

Min: Min is defined as the statistical minimum of a metric over the duration of a communication session. For example, if the end-to-end delay metric of an end-device within a communication session is reported $\mathrm{N}$ times by the RDS, then the Min end-to-end delay is the smallest of the $\mathrm{N}$ end-to-end delay metric values reported.

Max: Max is defined as the statistical maximum of a metric over the duration of a communication session. For example, if the end-to-end delay metric of an end-device within a communication session is reported $\mathrm{N}$ times by the RDS, then the Max End-to-End Delay is the largest of the $\mathrm{N}$ End-to-End Delay metric values reported.

7. Keeping Historical Data and Storage

It is evident from the document that the RAQMON MIB data need to be managed to optimize storage space. The large volume of data gathered in a communication session could be optimized for storage space by performing and storing only aggregated RAQMON metrics for history if required.

Examples of how such storage space optimization can be performed include:

1. Make data available through the MIB only at the end of a communication session, i.e., upon receipt of a NULL PDU. The aggregated data could be made available using the RAQMON MIB as Mean, Max, or Min entries and saved for historical purposes.

2. Use a time-based algorithm that aggregates data over a specific period of time within a communication session, thus requiring fewer entries, to reduce storage space requirements. For example, if an RDS sends data out every 10 seconds and the RRC updates the RAQMON MIB once every minute, for every 6 data points there would be one MIB entry.

Siddiqui, et al. Standards Track

[Page 29] 
3. Periodically delete historical data in accordance with an administrative policy. An example of such a policy would be to delete historical data older than 60 days. The implementation of such policies is left to the application developer's discretion, and their use is an operational concern.

8. Security Considerations

Security considerations associated with the RAQMON Framework are discussed below, and in greater detail in other RAQMON memos as is appropriate.

\subsection{The RAQMON Threat Model}

The vulnerabilities associated with the RAQMON Framework are a combination of those associated with the underlying layers up to the transport layer, and of possible exploits of RAQMON payload. Possible exploits of RAQMON payloads fall within these classes:

1. Unauthorized examination of sensitive information in the payload in transit.

2. Unauthorized modification of payload contents in transit, leading to:

a. Mis-identification of information from one RAQMON reporting session as belonging to another destined to the same RRC;

b. Mismapping of RAQMON sessions;

C. Various forms of session-level denial-of-service (DoS) attacks;

d. DoS through modification of RAQMON parameter values and statistics;

e. Invalid timestamps, leading to false interpretation of the monitored data, affecting call records information, and making difficult to place monitoring events in their appropriate temporal context.

3. Malformed payloads, permitting the exploitation of potential implementation weaknesses to compromise an RRC.

4. Unauthorized disclosure of sensitive data carried by application PDUs, leading to a breach of confidentiality. 
Consequently, threats based on unauthorized disclosure or modification of payloads or headers will have to be assumed.

\subsection{The RAQMON Security Requirements and Assumptions}

In order to preserve integrity of the RAQMON PDU against these threats, the RAQMON model must provide for cryptographically strong security services.

Consequently, the RAQMON framework must be able to provide for the following protections:

1. Authentication - the RRC should be able to verify that a RAQMON PDU was in fact originated by the RDS that claims to have sent it.

2. Privacy - Since RAQMON information includes identification of the parties participating in a communication session, the RAQMON framework should be able to provide for protection from eavesdropping, to prevent an unauthorized third party from gathering potentially sensitive information. This can be achieved by using various payload encryption technologies, such as Data Encryption Standard (DES), 3-DES, Advanced Encryption Standard (AES), etc.

3. Protection from DoS attacks directed at the RRC - RDSs send RAQMON reports as a side effect of an external event (for example, a phone call is being received). An attacker can try to overwhelm the RRC (or the network) by initiating a large number of events (i.e., calls) for the purpose of swamping the RRC with too many RAQMON PDUs.

To prevent DoS attacks against RRC, the RDS will send the first report for a session only after the session has been in progress for the five-second reporting interval. Sessions shorter than that should be stored in the RDS and will be reported only after that interval has expired.

\subsection{RAQMON Security Model}

The RAQMON architecture permits the use of multiple transport protocols. Most of these support a secure mode of operation. There are advantages to relying on the security provided at the transport protocol layer.

1. Transport-protocol-level security can generally protect the payload with end-to-end authentication, confidentiality, message integrity, and replay protection services. 
2. A good cryptographic security protocol always has an associated key management protocol. Use of transport protocol security relies on its key management and does not require development of another mechanism.

3. When transport protocol security is already enabled between the RDS and RRC, additional encryption and message authentication at the application level is avoided.

However, there are also shortcomings to be noted in relying on transport protocol security.

1. When session-level isolation of the different RAQMON sessions of an RDS-RRC pair is required, it will be necessary to open separate transport protocol instances. Such cases, however, may be rare.

2. Since security services are not provided by the RAQMON framework, the absence of transport or lower protocol security implies the absence of RAQMON security.

\section{Acknowledgements}

The authors would like to thank Andy Bierman, Alan Clark, Mahalingam Mani, Colin Perkins, Steve Waldbusser, Magnus Westerlund, and Itai Zilbershtein for the precious advices and real contributions brought to this document. The authors would also like to extend special thanks to Randy Presuhn, who reviewed this document for spelling and formatting purposes, and who provided a deep review of the technical content. We also would like to thank Bert Wijnen for the permanent coaching during the evolution of this document and the detailed review of its final versions. 
10. Normative References

[RFC791] Postel, J., "Internet Protocol", STD 5, RFC 791, September 1981 .

[RFC1812] Baker, F., "Requirements for IP Version 4 Routers", RFC 1812, June 1995.

[RFC2119] Bradner, S., "Key words for use in RFCs to Indicate Requirement Levels", BCP 14, RFC 2119, March 1997.

[RFC2474] Nichols, K., Blake, S., Baker, F., and D. Black, "Definition of the Differentiated Services Field (DS Field) in the IPv4 and IPv6 Headers", RFC 2474, December 1998 .

[RFC2475] Blake, S., Black, D., Carlson, M., Davies, E., Wang, Z., and W. Weiss, "An Architecture for Differentiated Service", RFC 2475, December 1998.

[RFC2679] Almes, G., Kalidindi, S., and M. Zekauskas, "A One-way Delay Metric for IPPM", RFC 2679, September 1999.

[RFC2680] Almes, G., Kalidindi, S., and M. Zekauskas, "A One-way Packet Loss Metric for IPPM", RFC 2680, september 1999.

[RFC2681] Almes, G., Kalidindi, S., and M. Zekauskas, "A Roundtrip Delay Metric for IPPM", RFC 2681, september 1999.

[RFC2819] Waldbusser, S., "Remote Network Monitoring Management Information Base", STD 59, RFC 2819, May 2000.

[RFC2959] Baugher, M., Strahm, B., and I. Suconick, "Real-Time Transport Protocol Management Information Base", RFC 2959, October 2000 .

[RFC3393] Demichelis, C. and P. Chimento, "IP Packet Delay Variation Metric for IP Performance Metrics (IPPM)", RFC 3393, November 2002 .

[RFC3416] Presuhn, R., Ed., "Version 2 of the Protocol Operations for the Simple Network Management Protocol (SNMP)", STD 62, RFC 3416, December 2002.

[RFC3550] Schulzrinne, H., Casner, S., Frederick, R., and V. Jacobson, "RTP: A Transport Protocol for Real-Time Applications", STD 64, RFC 3550, July 2003. 
[RFC3551] Schulzrinne, H. and S. Casner, "RTP Profile for Audio and Video Conferences with Minimal Control", STD 65, RFC 3551, July 2003 .

11. Informative References

[RFC1034] Mockapetris, P., "Domain names - concepts and facilities", STD 13, RFC 1034, November 1987.

[RFC1035] Mockapetris, P., "Domain names - implementation and specification", STD 13, RFC 1035, November 1987.

[RFC1123] Braden, R., "Requirements for Internet Hosts Application and Support", STD 3, RFC 1123, October 1989.

[RFC1305] Mills, D., "Network Time Protocol (Version 3) Specification, Implementation and Analysis", RFC 1305, March 1992.

[RFC1918] Rekhter, Y., Moskowitz, B., Karrenberg, D., de Groot, G., and E. Lear, "Address Allocation for Private Internets", BCP 5, RFC 1918, February 1996.

[RFC2914] Floyd, S., "Congestion Control Principles", BCP 41, RFC 2914, September 2000 .

[RFC3235] Senie, D., "Network Address Translator (NAT)-Friendly Application Design Guidelines", RFC 3235, January 2002.

[RFC3611] Friedman, T., Caceres, R., and A. Clark, "RTP Control Protocol Extended Reports (RTCP XR)", RFC 3611, November 2003 .

[RFC3729] Waldbusser, S., "Application Performance Measurement MIB", RFC 3729, March 2004.

[RFC4150] Dietz, R. and R. Cole, "Transport Performance Metrics MIB", RFC 4150, August 2005.

[RFC4711] Siddiqui, A., Romascanu, D., and E. Golovinsky, "Realtime Application Quality-of-Service Monitoring (RAQMON) MIB", RFC 4711, October 2006.

[RFC4712] Siddiqui, A., Romascanu, D., Golovinsky, E., Ramhman, M., and Y. Kim, "Transport Mappings for Real-time Application Quality-of-Service Monitoring (RAQMON) Protocol Data Unit (PDU)", RFC 4712, October 2006. 


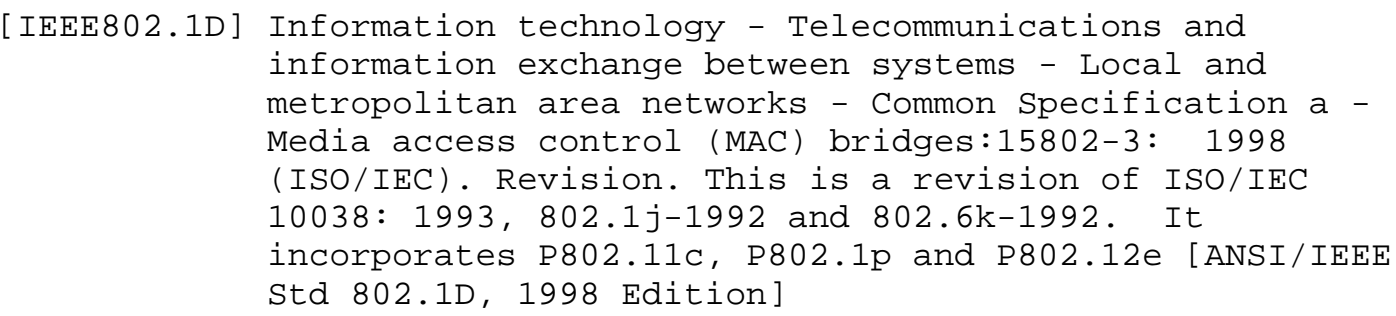


Full Copyright statement

Copyright (C) The Internet Society (2006).

This document is subject to the rights, licenses and restrictions contained in BCP 78, and except as set forth therein, the authors retain all their rights.

This document and the information contained herein are provided on an "AS IS" basis and THE CONTRIBUTOR, THE ORGANIZATION HE/SHE REPRESENTS OR IS SPONSORED BY (IF ANY), THE INTERNET SOCIETY AND THE INTERNET ENGINEERING TASK FORCE DISCLAIM ALL WARRANTIES, EXPRESS OR IMPLIED, INCLUDING BUT NOT LIMITED TO ANY WARRANTY THAT THE USE OF THE INFORMATION HEREIN WILL NOT INFRINGE ANY RIGHTS OR ANY IMPLIED WARRANTIES OF MERCHANTABILITY OR FITNESS FOR A PARTICULAR PURPOSE.

Intellectual Property

The IETF takes no position regarding the validity or scope of any Intellectual Property Rights or other rights that might be claimed to pertain to the implementation or use of the technology described in this document or the extent to which any license under such rights might or might not be available; nor does it represent that it has made any independent effort to identify any such rights. Information on the procedures with respect to rights in RFC documents can be found in BCP 78 and BCP 79 .

Copies of IPR disclosures made to the IETF Secretariat and any assurances of licenses to be made available, or the result of an attempt made to obtain a general license or permission for the use of such proprietary rights by implementers or users of this specification can be obtained from the IETF on-line IPR repository at http://www.ietf.org/ipr.

The IETF invites any interested party to bring to its attention any copyrights, patents or patent applications, or other proprietary rights that may cover technology that may be required to implement this standard. Please address the information to the IETF at ietf-ipreietf.org.

Acknowledgement

Funding for the RFC Editor function is provided by the IETF Administrative Support Activity (IASA) . 